\title{
Using Speech and Language Technology to Coach Reading
}

\author{
Patti Price \\ Formerly BravoBrava! LLC \\ Now PPRICE Speech and Language \\ 1-650-503-7053 \\ pjp@pprice.com
}

\author{
Luc Julia \\ BravoBrava! LLC \\ 32980 AlvaradoNiles Road, Suite 856 \\ Union City, CA 94587 \\ 1-510-477-0493 \\ Julia@bravobrava.com
}

\begin{abstract}
BravoBrava! is expanding the repertoire of commercial user interfaces by incorporating multimodal techniques combining traditional point and click interfaces with speech recognition, speech synthesis, and gesture recognition. One of these applications is software to help children read. While the child reads aloud, the computer keeps the child on track and offers feedback when the child has difficulty. The feedback can be as subtle as changing the text color for a well articulated phrase or as friendly as a cartoon character that talks. The computer is infinitely patient and can keep detailed records of the child's progress. The reading software is being commercialized by BravoBrava!'s spinoff company, SUP Inc.
\end{abstract}

Keywords: Reading, pedagogy

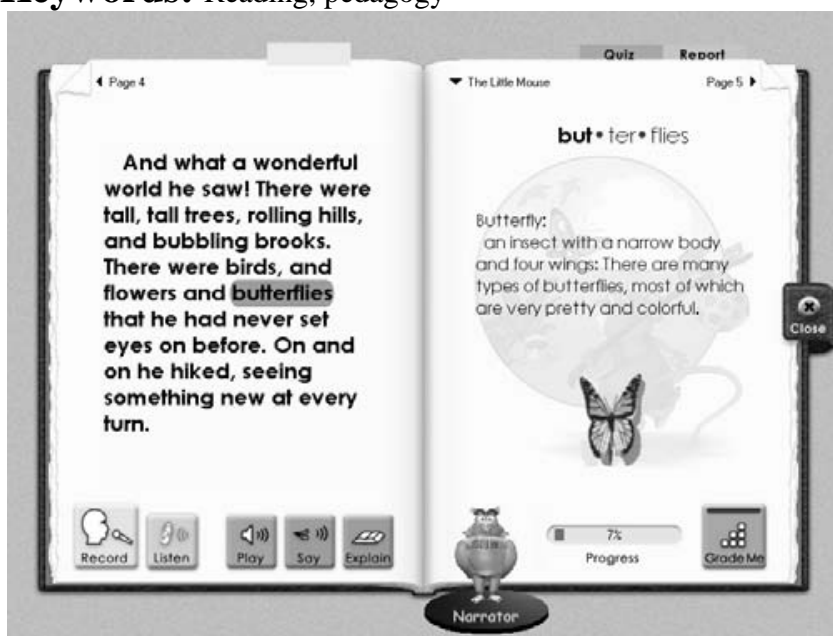

Figure 1. Here is an example from a sample story. The system tracks the reading and intervenes when help is needed. This screen shows a sample intervention if the child stumbles on the word 'butterflies'.

\section{INTRODUCTION}

Our vision is to use technology to provide a high-quality, low cost reading coach that delivers voice-activated reading instruction, practice, and assessment over electronic media. Reading is fundamental; it can also be fun. However, about $40 \%$ of mainstream 4th graders cannot read at the basic level. One of our country's critical needs is to improve reading performance for all children since their future, both individually and together, depends on literacy. Reading level predicts economic performance for both individuals and societies.

Beyond the basics, as reviewed in the recent report of the National Reading Panel (2000), engaging children in supported oral reading is the most valuable means toward building their reading proficiency. At present, however, the only means of giving children such practice is by finding a human adult who will sit with them and help them. However, technology can provide an automated reading coach to break through this bottleneck, so that every child in every school can get the support that she or he needs. This technology will help reduce the digital divide, and provide an unprecedented level of tracking data to leverage teachers' instruction and assessment efforts and to build the next generation of intervention techniques.

\section{SUP'S APPROACH}

SUP targets the stage at which children have learned the letter to sound rules but are still struggling to gain vocabulary and fluency. This stage has sometimes been called the transition from 'learning to read' to 'reading to learn' and comes just after the stage requiring explicit pronunciation and vocabulary tutoring, provided by Mostow's Project Listen at CMU and other reading software. At this stage in reading development, language skills are usually too poor to make traditional dictionaries much of a help. Dictionaries disrupt the child's focus on the text being read, offer too many definitions, and usually provide definitions that are harder for the child to read than the original text. Instead, SUP provides a generalized 'dictionary', the Reading Resource, to give immediate context specific help when needed, much the way a human reading coach might intervene. "SUPplementing" text with the Reading Resource, which includes word definitions, sample sentences, graphics and multimedia can create an engaging environment for learning. However, the immediacy and contextspecificity of this resource aims to maintain the child's focus on the material being read. Becoming engaged in the text itself is the 
real goal of reading. SUPplementation can make texts accessible to children that would otherwise be above their reading level, so that the content of the text can draw them into the desire to read. Vocabulary and grammatical knowledge grow with experience of more words in more contexts.

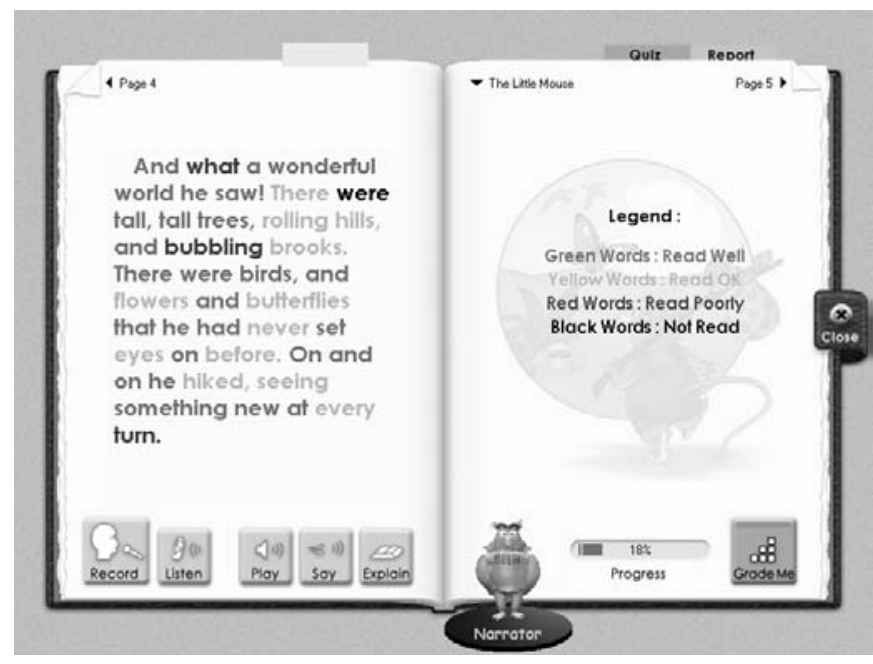

Figure 2. The screen shot above a sample after the child has asked for a display of how well the selection was read.

SUP has developed a modular architecture that allows for rapid reconfiguration:

- The current demonstration uses Microsoft speech recognition software. However, the architecture supports the use of other recognizers, and we have experimented with others for use on other platforms.

- The audio outputs can be from recorded waveforms or, for maximum flexibility, can use a text to speech synthesis system.

- Any text can flow through the system for reading practice.
Although explicit measures, such as a quiz to assess comprehension or vocabulary, can be included, several automatic measures are important by-products of use of the tool:

- Total number of words read by session, and which were fluent or not

- Words per minute as a function of time

- Level of the material read as a function of time

- Number of times intervention of the Reading Resource was used

- Number of times the child had the story read to him/her

- The actual recordings of what was read

\section{FUTURE DIRECTIONS}

Of course, such software will not help unless it gets into the hands of the children who need it. Therefore, an important strategy of the company is to support as many different platforms as possible to enable this goal. The modular architecture of the system facilitates transition to various platforms. Similarly, to be of maximal interest to the most children, we need as much appealing content as possible. In the area of human language technology, we hope such technology will evolve to automatically provide:

- Context-specific definitions

- Context-specific and child-specific synonyms

- Rewriting of complex phrases into simpler ones

Solving these problems completely is a major research project. However, we believe that with careful matchmaking between the technical possibilities and the real needs of beginning readers that progress can be made in the near term.

We hope that experience in the usability trials, just beginning, will also help us to plan similar products for adult language learners and learners of English as a second language. Reading is still the principal way that people learn information. In this information age, reading well is crucial to the individual and to society. 